\title{
Development and Implementation of the AIDA International Registry for Patients with Non- Infectious Scleritis
}

\author{
Francesca Della Casa · Antonio Vitale · Rosa Maria Pereira · Silvana Guerriero · Gaafar Ragab • \\ Giuseppe Lopalco · Marco Cattalini · Irene Mattioli · Paola Parronchi · Maria Pia Paroli · \\ Emanuela Del Giudice · Carla Gaggiano · Marília A. Dagostin • Valeria Albano · Mahmoud M. Soliman • \\ Sergio Colella · Giuseppe Nascimbeni · Jurgen Sota · Isabele P. B. Antonelli • Giovanni Alessio • \\ Valeria Caggiano · Abdurrahman Tufan • Rana Hussein Amin • Maria Tarsia • Mahmoud Ghanema • \\ Florenzo Iannone · Francesca Ricci · Francesco La Torre · Ewa Więsik-Szewczyk • Edoardo Conticini • \\ Stefano Gentileschi · Rosanna Dammacco • Rolando Cimaz • Bruno Frediani · Anna Abbruzzese • \\ Piero Ruscitti - Gian Marco Tosi · Heitor F. Giordano • Alessandro Conforti • Alberto Balistreri • \\ Donato Rigante $\cdot$ Luca Cantarini (D) C Claudia Fabiani
}

Received: December 13, 2021 / Accepted: January 12, 2022 / Published online: January 29, 2022

(C) The Author(s) 2022

\section{ABSTRACT}

Introduction: This article points out the design, methods, development and deployment of the international registry promoted by the AutoInflammatory Disease Alliance (AIDA) Network with the aim to define and assess

Luca Cantarini and Claudia Fabiani contributed equally.

F. Della Casa

Section of Clinical Immunology, Department of Translational Medical Sciences, University of Naples Federico II, Naples, Italy

A. Vitale · C. Gaggiano · J. Sota · V. Caggiano · M. Tarsia · E. Conticini - L. Cantarini ( $₫)$ Rheumatology Unit, Department of Medical Sciences, Surgery and Neurosciences, Research Center of Systemic Autoinflammatory Diseases and Behçet's Disease Clinics, University of Siena, Policlinico "Le Scotte", viale Bracci 16, 53100 Siena, Italy

e-mail: cantariniluca@hotmail.com

R. M. Pereira · M. A. Dagostin - I. P. B. Antonelli · H. F. Giordano

Rheumatology Division, Hospital das Clinicas (HCFMUSP), Faculdade de Medicina, Universidade de Sao Paulo, Sao Paulo, Brazil paediatric and adult patients with immunemediated scleritis.

Methods: This registry collects both retrospective and prospective real-world data from patients with non-infectious scleritis through the Research Electronic Data Capture (REDCap) tool and aims to promote knowledge and reallife evidence from patients enrolled worldwide; the registry also allows the collection of

\section{S. Guerriero · V. Albano - G. Alessio - R. Dammacco Department of Ophthalmology and Otolaryngology, University of Bari, Bari, Italy}

\section{G. Ragab · M. Ghanema}

Internal Medicine Department, Rheumatology and Clinical Immunology Unit, Faculty of Medicine, Cairo University, Giza, Egypt

\section{G. Ragab}

Faculty of Medicine, Newgiza University (NGU), Giza, Egypt

G. Lopalco · S. Colella · F. Iannone - A. Abbruzzese Rheumatology Unit, Department of Emergency and Organ Transplantation, University of Bari, Bari, Italy

M. Cattalini · F. Ricci

Pediatric Clinic, University of Brescia and Spedali

Civili di Brescia, Brescia, Italy 
standardised data, ensuring the highest levels of security and anonymity of patients' data and flexibility to change according to scientific acquisitions over time. The communication with other similar registries has been also ensured in order to pursue the sustainability of the project with respect to the adaptation of collected data to the most diverse research projects.

Results: Since the launch of the registry, 99 centres have been involved from 20 countries and four continents. Forty-eight of the centres have already obtained a formal approval from their local ethics committees. At present, the platform counts 259 users (95 principal investigators, 160 site investigators, 2 lead investigators, and 2 data managers); the platform collects baseline and follow-up data using 3683 fields organised into 13 instruments, including patient's demographics, history, symptoms,

I. Mattioli · P. Parronchi

Department of Experimental and Clinical Medicine,

University of Florence, Florence, Italy

M. P. Paroli

Uveitis Unit, Department of Sense Organs, Eye

Clinic, Sapienza University of Rome, Rome, Italy

E. Del Giudice

Department of Maternal Infantile and Urological

Sciences, Sapienza University of Rome, Polo

Pontino, Rome, Italy

M. M. Soliman · R. H. Amin

Opthalmology Department, Faculty of Medicine,

Cairo University, Giza, Egypt

G. Nascimbeni

Department of Ophthalmology, University Hospital

"Spedali Civili" of Brescia, Brescia, Italy

A. Tufan

Division of Rheumatology, Department of Internal

Medicine, Gazi University Faculty of Medicine,

Ankara, Turkey

F. La Torre

Pediatric Rheumatology Center, Department of

Pediatrics, Ospedale "Giovanni XXIII", AOU

Consorziale Policlinico, Bari, Italy trigger or risk factors, therapies and healthcare utilization.

Conclusions: The development of the AIDA International Registry for patients with noninfectious scleritis will allow solid research on this rare condition. Real-world evidence resulting from standardised real-life data will lead to the optimisation of routine clinical and therapeutic management, which are currently limited by the rarity of this ocular inflammatory condition.

Keywords: Autoinflammatory diseases; Clinical management; Inflammatory ocular diseases; Innovative biotechnologies; International registry; Personalised medicine; Precision medicine; Rare diseases

E. Więsik-Szewczyk

Department of Internal Medicine, Pulmonology, Allergy and Clinical Immunology, Central Clinical Hospital of the Ministry of National Defence, Military Institute of Medicine, Warsaw, Poland

S. Gentileschi · B. Frediani

Unit of Rheumatology, Azienda Ospedaliero-

Universitaria Senese, Siena, Italy

R. Cimaz

ASST G. Pini-CTO, Department of Clinical Sciences and Community Health, Research Center for Adult and Pediatric Rheumatic Diseases, University of Milan, Milan, Italy

P. Ruscitti · A. Conforti

Rheumatology Unit, Department of

Biotechnological and Applied Clinical Sciences, University of L'Aquila, L'Aquila, Italy

G. M. Tosi · C. Fabiani

Ophthalmology Unit, Department of Medicine, Surgery and Neurosciences, University of Siena, Siena, Italy

A. Balistreri

Bioengineering and Biomedical Data Science Lab, Department of Medical Biotechnologies, University of Siena, Siena, Italy 


\section{Key Summary Points}

The AutoInflammatory Disease Alliance (AIDA) International Registry for noninfectious scleritis is available and ready for data collection.

The registry will allow the scientific community to communicate, share information and actively participate in research projects.

Implementation and execution of a large international registry will overcome limitations owing to the low epidemiological burden of non-infectious scleritis.

Future solid evidence drawn from a wide range of standardised real-world data will corroborate results obtained from the few randomised clinical trials currently available.

\section{INTRODUCTION}

Non-infectious scleritis represents a group of conditions characterised by eye inflammation starting from the sclera and capable of spreading to adjacent ocular structures. It can occur in all age groups. Unlike episcleritis, which is a common condition usually causing mild discomfort with no risk of blindness [1], scleritis represents an inflammatory affection potentially leading to blindness. It manifests with chronic eye pain radiating to the face and scalp, worsening during the night, associated with decreased visual acuity and red eye. While both

\section{Rigante}

Department of Life Sciences and Global Health, Fondazione Policlinico Universitario A. Gemelli IRCCS, Rome, Italy

\section{Rigante}

Rare Diseases and Periodic Fevers Research Centre, Università Cattolica del Sacro Cuore, Rome, Italy the anterior and posterior parts of the sclera may be involved, anterior scleritis is more frequent and can be classified as diffuse, nodular, necrotizing with inflammation and necrotizing without inflammation [2]. Conversely, posterior scleritis may be diffuse or nodular. In several cases, scleritis is associated with rheumatoid arthritis, systemic lupus erythematosus [3], spondyloarthritis, and granulomatosis with polyangiitis, frequently representing their first manifestation [4]. In this context, a registry specifically dedicated to non-infectious scleritis is helpful to overcome limitations related to the rarity of this group of conditions and to the small numbers of patients available for research purposes. The reduced epidemiological burden of rare diseases leads to poor availability of patients for recruitment into clinical trials and to a consequent lack of knowledge about standardised care. This is even more challenging if considering the need for personalised medicine.

The AutoInflammatory Disease Alliance (AIDA) Network was born with the purpose of gathering a worldwide group of physicians and researchers interested in sharing knowledge, experience, information and different perceptions on the clinical, therapeutic and research approaches to autoinflammatory diseases and ocular non-infectious disorders, including scleritis. In addition, the focus of the AIDA project is the development and maintenance of international registries for patients with Behçet's disease, monogenic autoinflammatory diseases, Still's disease, Schnitzler's syndrome, periodic fever, aphthous stomatitis, pharyngitis and cervical adenitis (PFAPA) syndrome, non-infectious uveitis, vacuoles/E1 enzyme/X-linked autoinflammatory, somatic (VEXAS) syndrome and undifferentiated systemic autoinflammatory diseases (USAIDs). Each registry corresponds to a multicentre non-interventional observational cohort clinical study designed to investigate a specific inflammatory disease. Registries may be reached at the following webpage: https://aidanetwork.org/en/registries.

The main purpose of this paper is to point out the design, development and deployment of the AIDA International Registry specifically dedicated to patients with non-infectious scleritis unrelated to Behçet's disease (for which 
another specific AIDA registry is currently available).

\section{METHODS}

\section{Study Design}

As for other AIDA registries, this is a physiciandriven, population- and electronic-based registry accounting for a long-term observational study. It has been created for patients affected by non-infectious scleritis which may be idiopathic or associated with systemic immunemediated diseases. This international registry has been developed to collect both retrospective and prospective data. More specifically, the retrospective phase refers to real-world demographic, clinical, laboratory, instrumental and therapeutic data still available at the time of inclusion into the registry; prospective data are intended as clinical, therapeutic and socioeconomic information accrued starting from the time of enrolment. Prospective data must be updated at least once a year or rather at each follow-up visit, especially in the case of treatment change and posology adjustments.

No additional laboratory, clinical or instrumental investigations are required as a result of participation in the AIDA project and only data derived from standard routine management are required in the registry. None of the therapies prescribed to the patient must be determined by adherence to the study, as the registry is intended to "take a picture" of patient's clinical and therapeutic history. For these reasons, funds are not provided for the patient's enrolment and no further impact on national healthcare will be determined by participation in the AIDA project.

Any centre that manages patients with noninfectious scleritis could participate in the project. However, a mandatory prerequisite is obtaining approval from the local ethics committee and appointing a principal investigator, whose task is to coordinate the study locally, and site investigators responsible for the documentation and data entry. These represent preconditions to obtain credentials and access to the registry and share information.

\section{Registry Objectives}

The primary objective of this registry is to enrol a wide number of patients with non-infectious scleritis in order to carry out solid research leading to reliable results. Further aims include (i) assessment of clinical course and outcome of non-infectious scleritis in relation to the etiology (i.e. idiopathic versus forms associated with systemic diseases, distinguishing among the different diseases); (ii) evaluation of current diagnostic tools, grading of the disease and classification criteria in the light of the cuttingedge scientific acquisitions; (iii) to specifically taper the diagnostic and therapeutic approach according to the different etiologies; (iv) the identification of new clinical entities in the spectrum of idiopathic scleritis; (v) the assessment of paediatric-onset scleritis in relation to long-term sequelae and optimal management; (vi) identification of the role of different locoregional and intravitreal injections with respect to systemic therapies; (vii) evaluation of any difference in the scleritis course on the basis of demographic and clinical features; (vii) searching for any variable associated with the severity of scleritis in order to identify patients needing a more careful follow-up assessment or a more aggressive treatment early; (ix) identification of prognostic factors capable of the early identification of patients more likely to be responsive to different treatments; (x) understanding the behaviour and impact of inflammatory- and drug-related ocular complications on visual function and in the light of new diagnostic and therapeutic opportunities; (xi) identification of predisposing factors to noninfectious scleritis, severity of manifestations and response to treatments; (xii) description of the socioeconomic impact of the disease in relation to both the epidemiologic burden in different geographic contexts and benefits obtained from treatment approaches currently available; (xiii) behaviour of non-infectious scleritis during pregnancy and the breastfeeding period. Moreover, data collection will clarify the worldwide distribution of non-infectious scleritis, facilitating the recruitment of patients in future randomised controlled trials (RCTs). 
Certainly, further objectives will be considered in relation to unmet needs deriving from new scientific acquisitions in the future; for example, the new automatised data analysis tools, such as machine learning systems, represent an interesting approach to the management and analysis of a wide range of data. This approach will optimise clinical research based on real-world data from patients' registries and give a further boost to precision medicine implementation.

\section{Inclusion/Exclusion Criteria}

Inclusion criteria into this AIDA Registry are diagnosis of non-infectious scleritis made by an expert ophthalmologist after a careful exclusion of neoplastic and infectious disorders (e.g. herpes virus, Pseudomonas aeruginosa, Staphylococcus pneumoniae, tuberculosis, fungal infections). In addition, all patients enrolled have to provide their written informed consent after having been carefully informed about the project, its aims, privacy-related security according to local and/or country regulations, and the lack of any impact of the study on clinical practice. The patient has to be informed that they could refuse to enter the study or choose to withdraw from it at any time without any repercussions on their own clinical workflow. The parents, or a legally acceptable representative, and the adolescent subjects have to be willing and able to comply with the project requirements for the whole duration of the observation period.

The exclusions criteria are (i) the lack of informed consent/assent from the patient/legal guardians; (ii) identification of any infectious etiology explaining intraocular inflammation; (iii) diagnosis of Behçet's disease, for which a specific AIDA registry has been developed and activated.

\section{Online Data Collection}

Patients' information is collected using Research Electronic Data Capture (REDCap), an electronic data capture tool developed at the Vanderbilt University Medical Center (VUMC) and hosted at Virginia Commonwealth
University (Award Number UL1TR002649), which can also be used to develop patient registries. The software is distributed at no cost to members of the REDCap Consortium, which is a global collaboration of over 5300 diverse institutions from 143 countries that have installed the software and collaborate to provide support [5].

Investigators included into the AIDA project can log into the registry through the REDCap web interface, insert data on the pages (instruments) of the registry and then review (and eventually complete) their own information. Neither principal investigators nor site investigators are allowed to see information inserted by other centres. The electronic data entry system of the registry is in English.

The public website (https://aidanetwork.org/ en/) may be accessed by everyone who wants to learn about the AIDA Network and its significance, objectives and how to participate in the project. The registry website (https://sitbio.med. unisi.it/redcap/redcap_v11.4.3/index.php?pid= 29), instead, is hosted separately from the public website as a security measure. Data entry is password-protected, and the collected data are stored on a secure server in the University of Siena, Siena, Italy.

\section{Ethics}

Since the Ethics Committee of Azienda Ospedaliera Universitaria Senese, Siena, Italy, has provided the first national regulatory approval of the AIDA project (Ref. No. 14951) in June 2019, different centres from Europe, the Middle East, North Africa, South and North America have approved the project and joined AIDA Network.

The registry is run following the recommendations from the Declaration of Helsinki. Patients' participation in the registry is voluntary. After having received age-appropriate information sheets, patients enrolled (or their parents/caregivers) have to give their informed consent; minors aged 12 years or older are also required to provide their assent before inclusion in the study. Both patients and principal investigators may withdraw their consent for 
the use of data for statistical analyses at any time. If a patient withdraws their consent, no further data for that patient will be entered into the registry and, if specifically requested by the patient, all their prior data will be deleted from the registry soon after communication with the study promoter. Patients' data are kept in accordance with the EU General Data Protection Regulations (GDPR, 2016/679/EU) or its counterparts worldwide, on the processing of personal data and the protection of privacy [6].

Patients do not receive any honoraria or other types of remuneration for participation in the registry, and no economic impact on the national healthcare systems or insurance companies have to be pointed out.

\section{Statistical Analysis}

Statistical analysis will depend on the objectives to be achieved over time and on the type and number of data collected. However, in addition to descriptive statistics, the statistical plan will include correlations between groups and comparisons among subgroups; also, machine learning principles will be applied to implement the conventional data analysis.

\section{RESULTS}

The development and activation of this international registry is an important step to obtain solid scientific information from large cohorts of patients and different geographical contexts representative of the various environmental factors. Since its creation, this project has quickly reached a wide geographic coverage, reaching 20 countries (Algeria, Belgium, Brazil, Egypt, Germany, Ghana, Greece, Iran, Italy, Lebanon, Morocco, Poland, Portugal, Romania, Saudi Arabia, Spain, Taiwan, Turkey, the USA, Zimbabwe) in almost all continents. Ninetynine centres around the world have joined the AIDA project and 48 have already obtained approval from the local ethics committee. Currently, the AIDA Network counts 259 users (95 principal investigators, 160 site investigators, 2 lead investigators, 2 data managers).

\section{Registry Development}

Clinical variables constituting the registry have been chosen in order to carefully reconstruct the regular progression of a patient's clinical history along with their diagnostic, follow-up and therapeutic course. Additional items have been included with the aim to solve the current unmet needs concerning inflammatory ocular diseases. In particular, the registry is composed of 3683 common data elements (fields) organised into 13 instruments (forms).

The common data elements correspond to an equal number of items that describe the patient's demographics, medical history, laboratory features, association with other diseases, ocular involvement and associated systemic involvement, symptoms developing over time, comorbidities, cardiovascular risk, laboratory work-up, instrumental and ophthalmologic exams, pregnancies, past and current treatments, long-term clinical outcomes, short- and long-term response to treatments, response to different treatment strategies in terms of posology changes and drug combinations, and access to healthcare. The fields are organised with a branching mechanism of questions, which allows the appearance of some questions only if a patient's clinical history makes it necessary to answer them. Therefore, only a relatively small part of the 3683 fields will appear to the investigators, in relation to the specific patient's history.

Owing to the REDCap tool's flexibility, the registry may be continuously improved in accordance with new acquisitions derived from the international medical literature. Therefore, the number of variables included in the registry will change in the future in accordance with the need to answer future scientific questions and perform cutting-edge research.

Data elements, where appropriate, are shared with other AIDA registries; other bespoke data elements are specific to non-infectious uveitis and have been added to meet specific fields of this disease in terms of clinical manifestations, disease activity, possible complications, specific treatments, and different follow-up approaches planned for the prospective enrolment phase. In relation to this last point, longitudinal data 
Table 1 List of instruments (forms) included in the registry dedicated to patients with non-infectious scleritis, with the corresponding number of common data elements, the time points to which they refer and the number of mandatory fields included

\begin{tabular}{|c|c|c|c|}
\hline Instruments & Fields & $\begin{array}{l}\text { Retrospective/ } \\
\text { prospective phase }\end{array}$ & $\begin{array}{l}\text { No. of } \\
\text { mandatory } \\
\text { fields }\end{array}$ \\
\hline Demographics & 9 & Retrospective phase & 4 \\
\hline Consents & 4 & $\begin{array}{l}\text { Retrospective/ } \\
\text { prospective phase }\end{array}$ & 1 \\
\hline Diagnostic data and general information & 99 & Retrospective phase & 4 \\
\hline Features of scleritis and ocular history & 44 & Retrospective phase & 0 \\
\hline Cardiovascular risk & 19 & $\begin{array}{l}\text { Retrospective/ } \\
\text { prospective phase }\end{array}$ & 1 \\
\hline Data concerning fertility and pregnancy & 13 & $\begin{array}{l}\text { Retrospective/ } \\
\text { prospective phase }\end{array}$ & 1 \\
\hline Disease course and treatment during pregnancies & 61 & $\begin{array}{l}\text { Retrospective/ } \\
\text { prospective phase }\end{array}$ & 1 \\
\hline Treatments performed during patient's history_retrospective phase & 68 & Retrospective phase & 0 \\
\hline $\begin{array}{l}\text { Treatment with cDMARDs not associated with biologic agents- } \\
\text { retrospective phase }\end{array}$ & 370 & Retrospective phase & 6 \\
\hline $\begin{array}{l}\text { Treatment with small molecules not associated with biologic agents- } \\
\text { retrospective phase }\end{array}$ & 627 & Retrospective phase & 12 \\
\hline Treatment with biotechnological agents-retrospective phase & 967 & Retrospective phase & 17 \\
\hline $\begin{array}{l}\text { Ophthalmological assessment of each treatment performed during } \\
\text { patient's history_retrospective phase }\end{array}$ & 338 & Retrospective phase & 0 \\
\hline Follow-up visits-prospective phase & 1051 & Prospective phase & 51 \\
\hline
\end{tabular}

cDMARD conventional disease-modifying antirheumatic drug

are captured through a specific follow-up instrument including the ophthalmologic assessment, evaluation of the systemic disease course (in cases with a concomitant systemic inflammatory condition) and treatments performed in the previous observation period. Of note, this registry may also communicate with other existing and/or future registries with similar purposes. Indeed, merging data among different registries will enhance the sustainability of this project, which will be essential to adapt collected data to answer different research questions and to assess this inflammatory ocular disease from different perspectives.
Both the retrospective and the prospective phases require the compilation of specific data drawn from the ophthalmologic examination including any instrumental examinations aimed at assessing morphological and functional ocular parameters according to the clinical picture. In addition, the following items are required: the best corrected visual acuity (BCVA), the scleritis grading system proposed by Sen et al.; the McCluskey scoring system for monitoring scleritis response to treatment; ocular ultrasonography and orbital magnetic resonance imaging; optical coherence tomography (OCT) evaluation; the occurrence of any 
ocular complication or concomitant affection such as peripheral ulcerative keratitis and/or uveitis. Also, the ASUWOG fluorescein angiography scoring system and the ASUWOG indocyanine green angiographic scoring system are needed if required by specific concomitant ocular affections [7-11]. Table 1 provides a full list of instruments constituting the registry and the phases to which they refer.

\section{DISCUSSION}

During recent years, electronics and informatics bioengineering have allowed the increasing use of online platforms aimed at international collaboration and real-world data collection, to quickly get essential information for scientific purposes in many medical fields. This is even truer in the field of rare diseases characterised by a reduced epidemiological burden and enormous difficulties with patient enrolment for clinical studies. In the past decades, case reports, case series and small retrospective studies were the only studies conducted on rare diseases because of the small number of patients and difficulties experienced in sharing data. The development of online platforms and registries has allowed the resolution of these issues, enabling the collaboration of international centres worldwide. In parallel, the number of patients enrolled in clinical studies has gradually expanded and has made it possible to carry out studies based on a sufficient number of patients and even RCTs. Examples of this new scientific approach are represented by online registries dedicated to rare tumours in children (PARTNER and EXPeRT), rare endocrine conditions (EuRRECa), and the Childhood Arthritis and Rheumatology Research Alliance registry (CARRA) for better assessing current treatment strategies for juvenile-onset arthritis [12-15].

In this context, real-world data have recently emerged as a pivotal area of interest for researchers, as age at enrolment, patient's behaviour, co-occurring treatments and environmental factors may represent essential elements influencing disease course, prognosis and response to treatment. Actually, while these elements often appear among exclusion criteria of RCTs, they are part and parcel of the assessment in real-life studies. This leads to more powerful insights regarding the application of results in routine clinical practice, as real-world settings are neither influenced nor limited by protocol boundaries or the exclusion of specific borderline cohorts of patients. In this scenario, the AIDA project has taken charge of the implementation and diffusion of the first online registry for patients with non-infectious scleritis. The launch of this project may facilitate the communication among referral centres and finalise international research projects also in the field of immune-mediated scleritis, which represents both a rare condition and an extremely varied clinical entity that must be adequately studied on the basis of large enough cohorts of patients.

As for other immune-mediated diseases, the management of non-infectious scleritis requires the collaboration of different specialties that must necessarily communicate with each other, including ophthalmologists, rheumatologists, geneticists, immunologists, gastroenterologists, dermatologists, internal medicine physicians, and paediatricians. For this reason, a registry accessible worldwide may overcome the divisions and fragmentation of knowledge on such conditions and can also gather a wide range of specialties ready to provide their own contribution to research.

The AIDA project has already achieved the goal of developing an online platform dedicated to autoinflammatory diseases and inflammatory ocular diseases; this platform represents a virtual forum where the scientific community may easily get in touch, share experience, and actively participate in international research projects, without worrying about the limited numbers of patients or wasting time to specifically contact other centres. Therefore, the implementation and execution of this large international registry may finally overcome the limitations of smaller individual studies [16], providing valuable and generalizable real-world evidence to apply in the routine management of patients.

The availability of large amounts of data from this international registry will make it possible to characterise new clinical entities, 
possibly reducing the percentage of idiopathic forms of scleritis. In this regard, the cluster analysis and the application of machine learning systems may bring about the identification of specific subgroups of patients with similar features accounting for new clinical entities.

Furthermore, a large-scale, long-term patient registry may provide additional evidence on the behaviour of non-infectious scleritis in the short and long term, also taking into account geographical distribution, different treatments and any features bringing together specific subgroups of patients who may behave differently in terms of prognosis and response to treatments. Additionally, in an era when the need for personalised medicine aims at tailoring the daily care and treatments on the basis of patient's specific features, future research must aspire to identify clinical variables useful for predicting the clinical course of the disease, and consequently identifying patients requiring a more intensive treatment and preventing the onset of severe manifestations and/or the development of complications.

All the objectives of this registry are even more pivotal in the light of recent diagnostic and treatment acquisitions. These aspects should be implemented, thereby widening as much as possible the number of patients included, who should not be necessarily severe and complicated. The registry may also provide an invaluable source of data to better understand the behaviour of ocular inflammatory disorders during and after pregnancy. A further outcome of this registry concerns the application of currently used clinimetric tools and development of new instruments capable of assessing disease severity as well as the short- and long-term prognosis. The worldwide distribution of patients enrolled will allow the evaluation of both accuracy and specificity of these clinimetric tools according to the different geographical contexts and patient's specific systemic disease as well as treatments used. The prospective phase of the project will also provide more information on the socioeconomic impact of the disease and eventual benefits that national health systems can obtain from treatments used.
The AIDA Registry for patients with non-infectious scleritis has the usual limitations of observational research and registries. Of note, entering data into the registry requires time and attention, especially when a patient's medical history is quite complex, such as when they have undergone multiple treatment approaches and many posology changes, or when many relapses have occurred. Therefore, while entering prospective data from follow-up visits takes 10-15 min, entering retrospective data from a particularly complex case can take many hours. In addition, some ophthalmologic examinations required in the registry may be highly specialistic for non-ophthalmologist investigators. In this regard, close collaboration with the referral ophthalmologist may be recommended. Also note that the investigators are under no obligation to consecutively enrol patients presenting to their centre because of non-infectious scleritis; this may cause unintended selection bias. Nevertheless, beyond its limitations, this is the first international registry for patients with non-infectious scleritis: the worldwide geographical basis will guarantee the achievement of all objectives fixed and explained in this paper. Moreover, an electronic system for collecting patient-reported data (AIDA for patients) is about to be developed; this will minimize missing values and resolve many of the current limitations of the registry.

In conclusion, the development of this AIDA International Registry dedicated to patients with non-infectious scleritis will facilitate the sharing of data and experience among a large number of centres managing scleritis worldwide. The collection of standardised real-world data will promote international multicentre collaborative research projects for both retrospective and prospective studies. The use of reallife data obtained from this registry will allow the use of real-world evidence for the daily clinical management and therapeutic approach of patients with non-infectious scleritis. In addition, future prospective RCTs and international consultation, along with the potential active involvement of patients and their families may represent extraordinary results of this project. The next step will include a further dissemination of the network, the inclusion of 
all the stakeholders possibly interested in the project, and the start of the first studies based on the real-world data already collected.

\section{ACKNOWLEDGEMENTS}

Funding. No funding or sponsorship was received for this study or publication this article.

Authorship. All named authors meet the International Committee of Medical Journal Editors (ICMJE) criteria for authorship for this article, take responsibility for the integrity of the work as a whole, and have given their approval for this version to be published.

Author Contributions. Francesca Della Casa wrote the first draft of the manuscript; Antonio Vitale, conceived and designed the study and revised the draft of the manuscript; Claudia Fabiani, conceived and designed the study and the non-infectious scleritis registry; Donato Rigante, revised the draft of the manuscript; Luca Cantarini, conceived and designed the study and acts as AIDA Registries Coordinator; Rosa Maria Pereira, Silvana Guerriero, Gaafar Ragab, Giuseppe Lopalco, Marco Cattalini, Irene Mattioli, Paola Parronchi, Maria Pia Paroli, Emanuela Del Giudice, Carla Gaggiano, Marília A. Dagostin, Valeria Albano, Mahmoud M Soliman, Sergio Colella, Giuseppe Nascimbeni, Jurgen Sota, Isabele P. B. Antonelli, Giovanni Alessio, were involved in data recruitment in the registry dedicated to patients with non-infectious scleritis; Valeria Caggiano, Abdurrahman Tufan, Rana Hussein Amin, Maria Tarsia, Mahmoud Ghanema, Florenzo Iannone, Francesca Ricci, Francesco La Torre, Ewa WięsikSzewczyk, Edoardo Conticini, Stefano Gentileschi, Rosanna Dammacco, Rolando Cimaz, Bruno Frediani, Anna Abbruzzese, Piero Ruscitti, Gian Marco Tosi, Heitor F. Giordano, Alessandro Conforti, were included in the authorship as investigators from to the three top contributor centres for any of the other eight AIDA registries; Alberto Balistreri, is the bioengineer involved in the technical management of the platform and registries. Authorship has been established based on the number of data recruited in the AIDA registries on 8 December 2021.

Compliance with Ethics Guidelines. Ethics Committee of Azienda Ospedaliera Universitaria Senese, Siena, Italy, has provided the first regulatory approval of the AIDA project (Ref. No. 14951) in June 2019. Ninety-nine centres around the world have joined the AIDA project and 48 have already obtained approval from the local ethics committee. The registry is run following the recommendations from the Declaration of Helsinki of 1964 and its later amendments.

Disclosures. Francesca Della Casa, Antonio Vitale, Rosa Maria Pereira, Silvana Guerriero, Gaafar Ragab, Giuseppe Lopalco, Marco Cattalini, Irene Mattioli, Paola Parronchi, Maria Pia Paroli, Emanuela Del Giudice, Carla Gaggiano, Marília A. Dagostin, Valeria Albano, Mahmoud M Soliman, Sergio Colella, Giuseppe Nascimbeni, Jurgen Sota, Isabele P. B. Antonelli, Giovanni Alessio, Valeria Caggiano, Abdurrahman Tufan, Rana Hussein Amin, Maria Tarsia, Mahmoud Ghanema, Florenzo Iannone, Francesca Ricci, Francesco La Torre, Ewa Więsik-Szewczyk, Edoardo Conticini, Stefano Gentileschi, Rosanna Dammacco, Rolando Cimaz, Bruno Frediani, Anna Abbruzzese, Piero Ruscitti, Gian Marco Tosi, Heitor F. Giordano, Alessandro Conforti, Alberto Balistreri, Donato Rigante, Luca Cantarini, Claudia Fabiani have nothing to disclose.

Data Availability. The datasets generated during and/or analyzed during the current study are available from the corresponding author on reasonable request.

Open Access. This article is licensed under a Creative Commons Attribution-NonCommercial 4.0 International License, which permits any non-commercial use, sharing, adaptation, distribution and reproduction in any medium or format, as long as you give appropriate credit to the original author(s) and the source, provide a link to the Creative Commons licence, and 
indicate if changes were made. The images or other third party material in this article are included in the article's Creative Commons licence, unless indicated otherwise in a credit line to the material. If material is not included in the article's Creative Commons licence and your intended use is not permitted by statutory regulation or exceeds the permitted use, you will need to obtain permission directly from the copyright holder. To view a copy of this licence, visit http://creativecommons.org/licenses/by$\mathrm{nc} / 4.0 /$.

\section{REFERENCES}

1. McCluskey P, Powell RJ. The eye in systemic inflammatory diseases. Lancet. 2004;364(9451): 2125-33. https://doi.org/10.1016/S01406736(04)17554-5.

2. Wakefield D, Di Girolamo N, Thurau S, Wildner G, McCluskey P. Scleritis: challenges in immunopathogenesis and treatment. Discov Med. 2013;16(88):153-7.

3. Kuhn A, Landmann A. The classification and diagnosis of cutaneous lupus erythematosus. J Autoimmun. 2014;48-49:14-9. https://doi.org/10.1016/j. jaut.2014.01.021.

4. Generali E, Cantarini L, Selmi C. Ocular involvement in systemic autoimmune diseases. Clin Rev Allergy Immunol. 2015;49(3):263-70. https://doi. org/10.1007/s12016-015-8518-3.

5. Harris PA, Taylor R, Thielke R, Payne J, Gonzalez N, Conde JG. Research electronic data capture (REDCap) - a metadata-driven methodology and workflow process for providing translational research informatics support. J Biomed Inform. 2009;42(2): 377-81. https://doi.org/10.1016/j.jbi.2008.08.010.

6. Regulation (EU) $2016 / 679$ of the European Parliament and of the Council of 27 April 2016 on the protection of natural persons with regard to the processing of personal data and on the free movement of such data, and repealing Directive 95/46/ EC (General Data Protection Regulation-Text with EEA relevance). https://eur-lex.europa.eu/eli/reg/ 2016/679/oj. Accessed 15 Nov 2021.

7. Watson PG, Hayreh SS. Scleritis and episcleritis. Br J Ophthalmol. 1976;60(3):163-91. https://doi.org/ 10.1136/bjo.60.3.163.
8. Chylack LT Jr, Wolfe JK, Singer DM, et al. The Lens Opacities Classification System III. The Longitudinal Study of Cataract Study Group. Arch Ophthalmol. 1993;111(6):831-6. https://doi.org/10.1001/ archopht.1993.01090060119035.

9. Sen HN, Sangave AA, Goldstein DA, et al. A standardized grading system for scleritis. Ophthalmology. 2011;118(4):768-71. https://doi.org/10.1016/j. ophtha.2010.08.027.

10. Tugal-Tutkun I, Herbort CP, Khairallah M, Angiography Scoring for Uveitis Working Group (ASUWOG). Scoring of dual fluorescein and ICG inflammatory angiographic signs for the grading of posterior segment inflammation (dual fluorescein and ICG angiographic scoring system for uveitis). Int Ophthalmol. 2010;30(5):539-52. https://doi. org/10.1007/s10792-008-9263-x.

11. McCluskey P, Wakefield D. Prediction of response to treatment in patients with scleritis using a standardised scoring system. Aust N Z J Ophthalmol. 1991;19(3):211-5. https://doi.org/10.1111/j.14429071.1991.tb00663.x.

12. Orbach D, Ferrari A, Schneider DT, et al. The European Paediatric Rare Tumours NetworkEuropean Registry (PARTNER) project for very rare tumors in children. Pediatr Blood Cancer. 2021;68(Suppl 4): e29072. https://doi.org/10.1002/ pbc. 29072 .

13. Ferrari A, Schneider DT, Bisogno G, et al. Facing the challenges of very rare tumors of pediatric age: The European Cooperative Study Group for Pediatric Rare Tumors (EXPeRT) background, goals, and achievements. Pediatr Blood Cancer. 2021;68(Suppl 4): e28993. https://doi.org/10.1002/pbc.28993.

14. Ali SR, Bryce J, Smythe C, et al. Supporting international networks through platforms for standardised data collection-the European Registries for Rare Endocrine Conditions (EuRRECa) model. Endocrine. 2021;71(3):555-60. https://doi.org/10.1007/ s12020-021-02617-0.

15. Beukelman T, Kimura Y, Ilowite NT, et al. The new Childhood Arthritis and Rheumatology Research Alliance (CARRA) registry: design, rationale, and characteristics of patients enrolled in the first 12 months. Pediatr Rheumatol Online J. 2017;17(15): 30. https://doi.org/10.1186/s12969-017-0160-6.

16. Kempf L, Goldsmith JC, Temple R. Challenges of developing and conducting clinical trials in rare disorders. Am J Med Genet A. 2018;176(4):773-83. https://doi.org/10.1002/ajmg.a.38413. 